$62^{\text {ème }}$ Congrès de la SFCO, 02004 (2014)

DOI: $10.1051 /$ sfco/20146202004

(C) Owned by the authors, published by EDP Sciences, 2014

\title{
Avulsion des quatre dents de sagesse sous anesthésie locale : étude pharmacocinétique et effets cardiovasculaires.
}

\author{
Noel-Furon L', Mauprivez C ${ }^{1,2}$, Miric T', Vanscoor D', Hoizey G ${ }^{3}$, Lefevre B ${ }^{1}$ \\ 1 Unité de chirurgie orale - Pôle d'odontologie, CHU de REIMS, 45 rue Cognacq-Jay, 51100, Reims, FRANCE \\ 2 Service d'Odontologie, Groupe Hospitalier Pitié-Salpêtrière, 47-83 Boulevard de l'Hôpital, 75013, Paris, FRANCE \\ 3 Laboratoire de Pharmacologie et Toxicologie, CHU de REIMS, 45 rue Cognacq-Jay, 51100, Reims, FRANCE
}

\section{Introduction}

L'avulsion de plusieurs dents de sagesse en 1 temps opératoire est un acte chirurgical, le plus souvent, réalisé sous anesthésie générale (AG) dans le cadre d'une hospitalisation. (Trost et coll., 2007). Le développement de la chirurgie ambulatoire a permis de développer des protocoles anesthésiques améliorant la rapidité et la qualité du réveil anesthésique (SFAR, 2009). Mais l'emploi d'agents morphiniques et d'hypnotiques lors de l'AG expose toujours à la survenue d'évènements indésirables (nausées, vomissements, sédation excessive) durant la période postopératoire et peuvent être à l'origine d'une prolongation de la durée d'hospitalisation. II n'existe pas de données scientifiquement établies pour recommander une anesthésie générale de façon systématique en vue de réaliser l'extraction de plusieurs dents de sagesse (HAS, 2005). L'anesthésie locale (AL) seule ou associée à une sédation par voie intraveineuse peut être proposée comme une alternative à l'AG pour pratiquer ce geste. Cette modalité anesthésique implique l'utilisation d'une dose importante d'anesthésique local et soulève le problème d'un éventuel risque de surdosage. Le but de cette étude pilote est d'évaluer le profil pharmacocinétique de l'articaine après infiltration sous muqueuse d'une dose de $476 \mathrm{mg}$ de chlorhydrate d'articaïne (correspondant à 7 cartouches de $1,7 \mathrm{~mL}$ de chlorhydrate d'articaïne à $4 \%$, à $1: 200.000 \mathrm{e}$ d'adrénaline) et des effets cardiovasculaires associés.

Matériel et méthodes

7 patients âgés de 13 à 40 ans sont inclus, 5 hommes et 2 femmes. 6 patients sont retenus pour recevoir deux séries d'injections de 3,5 cartouches espacées de 30 minutes chacune. 1 patient répondant aux mêmes critères est retenu pour recevoir les 7 cartouches en injection unique. Des prélèvements sanguins sont réalisés dans les deux situations à des intervalles réguliers afin de doser la concentration plasmatique de l'articaïne. Une surveillance des constantes physiologiques cardio-circulatoires (tension artérielle, fréquence cardiaque et saturation en oxygène) est effectuée parallèlement durant tout le temps opératoire.

\section{Résultats}

L'articaine présente une pharmacocinétique linéaire d'ordre 2 en cas d'infiltrations sous muqueuse pour une anesthésie dentaire. En cas d'injection réitérée, le délai d'apparition du 1er pic plasmatique d'articaïne (Tmax1) est observé à 12,5 $\pm 6,9$ min avec une concentration plasmatique maximale (Cmax 1 ) de 1207 $\pm 346 \mu \mathrm{g} / \mathrm{L}$. Après la 2ème série d'injections, le Tmax2 est à 10,8 $\pm 3,8$ min et la Cmax2 à $1660 \pm 483 \mu \mathrm{g} / \mathrm{L}$. La phase d'élimination se caractérise par une demi-vie d'élimination (t1/2) de $32,5 \pm 10,8$ min et une clairance totale (CIT) de 106,3 $\pm 46,2 \mathrm{mg} . \mathrm{h} / \mathrm{L}$. En cas d'injection unique des 7 cartouches, le délai d'apparition du pic plasmatique d'articaine est de 30 minutes pour une durée d'injection de $12 \mathrm{~min}$. La Cmax est de $2311 \mu \mathrm{g} / \mathrm{L}$. La phase de décroissance est biphasique et suit un modèle bi-compartimental avec une demi-vie de distribution (t1/2a) de 12 min et une demi-vie d'élimination (t1/2a) de 44 min. La dose d'articaïne nécessaire pour pratiquer l'avulsion des 4 dents de sagesse rapportée au poids de chaque patient étudié donne une valeur moyenne de $6,95 \pm 1,14 \mathrm{mg} / \mathrm{Kg}$. Sur les 7 patients évalués, la dose maximale recommandée de l'articaïne $(7 \mathrm{mg} / \mathrm{Kg})$ est dépassée 4 fois. Aucune manifestation clinique de toxicité systémique, ni aucune perturbation hémodynamique majeure n'est observée durant la période opératoire.

\section{Discussion}

Malgré l'emploi de doses élevées d'articaïne (476 mg de chlorhydrate d'articaïne), aucune manifestation toxique ou de surdosage n'est rapportée. Ces résultats préliminaires confirment que l'articaïne possède un index thérapeutique important. Dans l'indication de l'avulsion de 4 dents de sagesse en 1 temps chirurgical, l'anesthésie locale seule ou associée à une sédation intraveineuse peut être proposée comme une alternative à l'anesthésie générale.

NOEL-FURON Laurence laurence.furon@wanadoo.fr

This is an Open Access article distributed under the terms of the Creative Commons Attribution License 4.0, which permits unrestricted use, distribution, and reproduction in any medium, provided the original work is properly cited. 